\title{
Acrometastases: The nasty alter ego of fingertip infections
}

\author{
Collin Seng Kim Looi, Manohar A/L Arumugam \\ Looi CSK, Arumugam M. Acrometastases: The nasty alter ego of fingertip infections. Malays Fam Physician. 2021;16(2);86-89. \\ https://doi.org/10.51866/cr1059
}

Keywords:

Acrometastases, breast

carcinoma

\section{Authors:}

\section{Collin Looi Seng Kim \\ (Corresponding author) \\ MBBS (Malaysia) MRCS (Ireland) \\ MS Ortho (UPM) \\ Orthopaedic Surgeon \\ Hand and Microsurgery Division \\ Department of Orthopaedic Surgery \\ Universiti Putra Malaysia \\ Email: collinlooi@gmail.com}

Manohar A/L Arumugam

MBBS (UM) MS Ortho (UM)

Consultant Orthopaedic, Hand

\& Microsurgeon, Hand and

Microsurgery Division

Department of Orthopaedic Surgery

Universiti Putra Malaysia

\begin{abstract}
Fingertip infections are commonly diagnosed in primary care. There is a serious condition, acrometastases, that is often mistaken for these infections. Acrometastases are defined as metastases located distal to the elbow or knee. We present a case of a malignant phyllodes tumour with acrometastases to the distal phalanx of the left middle finger that was misdiagnosed as a fingertip infection on 2 separate occasions, highlighting the need for vigilance regarding acrometastases.
\end{abstract}

\section{Introduction}

Acrometastases are rare, accounting for $0.1 \%$ of all metastases. Breast malignancies contribute $10.2 \%$ of these rare lesions. ${ }^{2}$ Phyllodes tumours are uncommon fibro-epithelial lesions that account for $0.3 \%$ to $0.5 \%$ of all breast tumours. ${ }^{11}$ While they are predominantly benign, malignant variants exist, with $10 \%$ presenting with metastases. ${ }^{2}$ Due to their rarity and manner of presentation, patients are commonly misdiagnosed. Poor prognosis and imminent death are associated with this condition, hence the need for a high index of suspicion to ensure prompt referral for palliative treatment.

\section{Case Report}

A 58-year-old female presented with left middle fingertip swelling and pain lasting 3 weeks, associated with a low-grade fever for 8 days that had gradually worsened. A general practitioner had initially diagnosed her with a fingertip infection and managed her with oral antibiotics. However, her symptoms failed to abate, leading her to seek further treatment at another facility. A more thorough patient history revealed that she was diagnosed with a right cystosarcoma phyllodes breast tumour with lung metastases a year prior and had undergone a right mastectomy. Six cycles of radiotherapy had been completed a month before the onset of her presenting symptoms. A surveillance CT scan of the thorax, abdomen, and pelvis, taken 4 days before presentation, revealed worsening lung metastases. She was clinically gaunt with a low-grade fever. Chest examination revealed a well-healed right mastectomy scar. Right submandibular lymphadenopathy was elicited.

There was an ulcerated fungating mass measuring approximately $3 \times 3 \times 3 \mathrm{~cm}$ at the tip of the left middle finger that was hard in consistency, as well as tender and warm on palpation (Figure 1). The range of motion at the distal interphalangeal joint was severely limited. Examination of all other fingers of the left hand was otherwise unremarkable. Her total white cell count and inflammatory markers were within normal limits. However, her chest radiograph showed cannonball lesions over the right mid-zone (Figure 2), while those of the left hand showed lytic destruction of the distal phalanx of the middle finger with a surrounding ill-defined radiolucent swelling (Figure 3). The adjacent joint surface was preserved. Despite red-flag signs suggestive of acrometastasis to the hand, the patient was misdiagnosed again by another physician as having a left middle fingertip abscess complicated with osteomyelitis of the left middle finger distal phalanx by another physician. However, due to the severity of her condition, an orthopaedic consult was sought. After a thorough review of her history, clinical findings, and investigations, her diagnosis was revised to acrometastasis of the distal phalanx of the left middle finger.

An MRI of the left-hand revealed an area with a hyperintense signal indicative of marrow replacement, with an expansile soft tissue mass at the distal phalanx of the middle finger in addition to a suspicious lesion at the proximal phalanx of the ring finger. The patient underwent disarticulation of the left middle finger distal inter-phalangeal joint and was subsequently referred to the oncology centre that had previously cared for her to resume palliative care. Unfortunately, she passed away a few weeks later due to deterioration. 

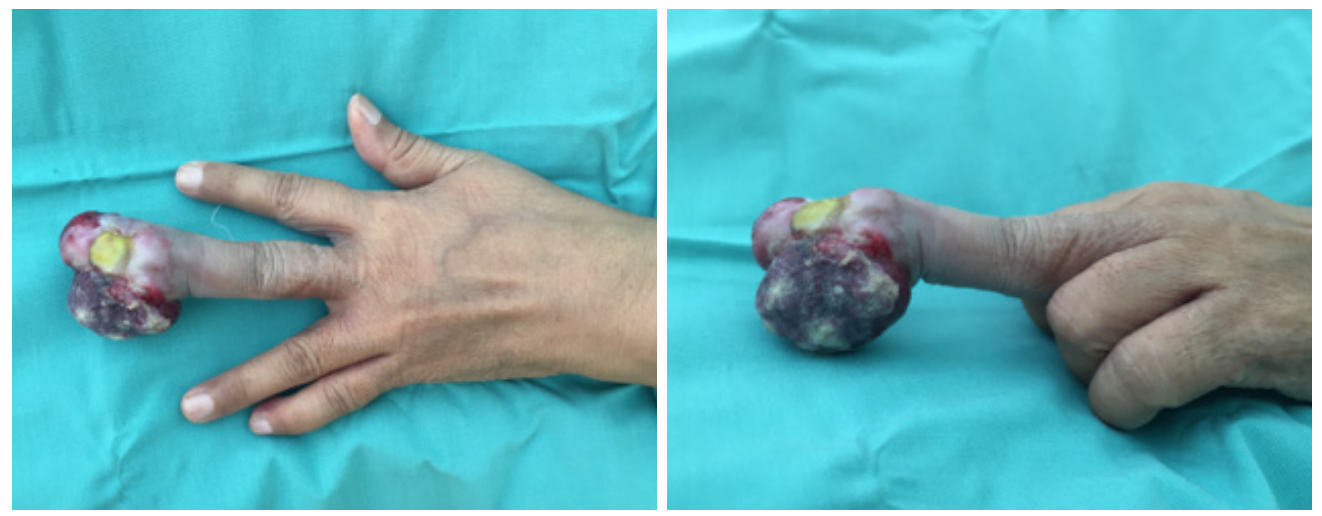

Figure 1: An ulcerating and fungating lesion at the distal phalanx of the left middle finger, measuring approximately $3 \times 3 \times 3 \mathrm{~cm}$ in size

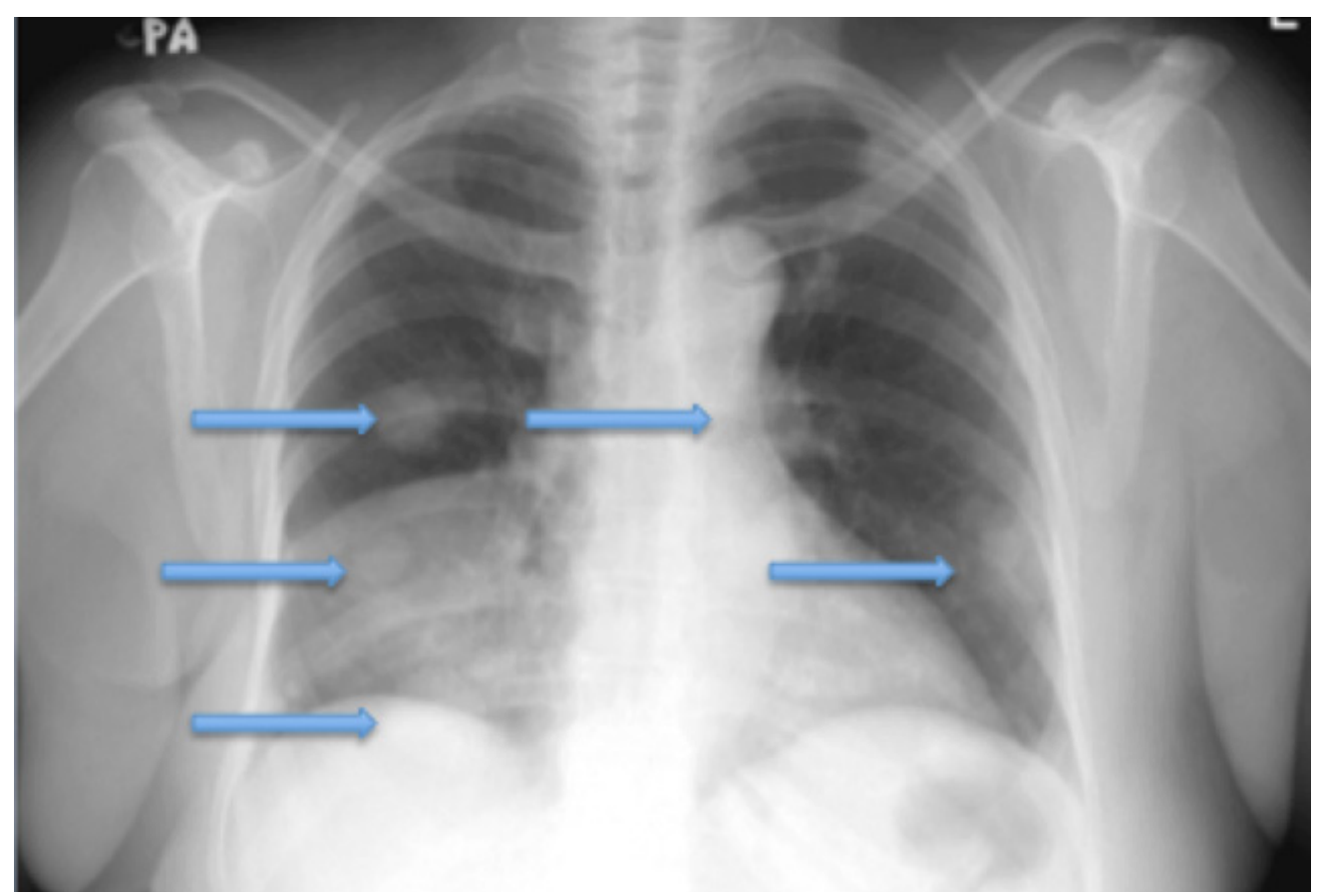

Figure 2: Chest radiograph showing multiple well-defined round opacities (indicated by arrows) within the right and left lung fields depicting cannonball lesions indicative of metastases to the lungs

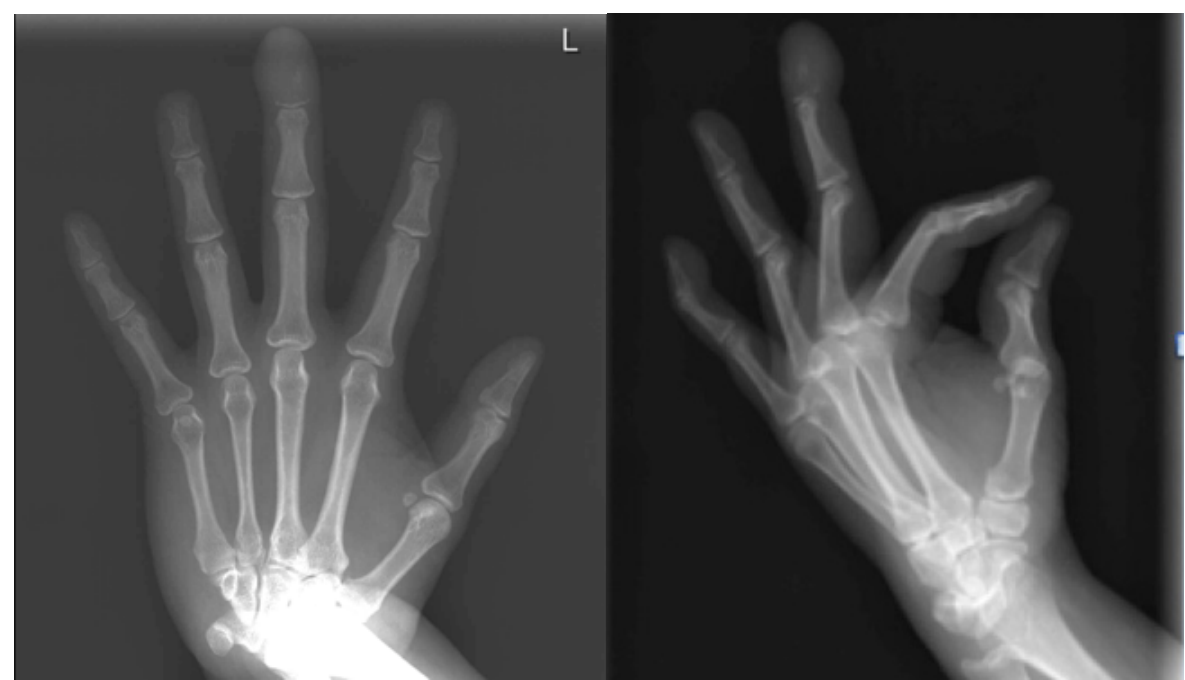

Figure 3: Permeative lytic destruction of the left middle finger distal phalanx with surrounding soft tissue swelling seen on both PA and oblique radiographs of the left hand 


\section{Discussion}

Skeletal metastases are known to occur in $20-30 \%$ of all malignancies. Acrometastases are defined as metastases located distal to the elbow or knee that are extremely rare, accounting for $0.1 \%$ of all metastases. ${ }^{1}$ These are usually found in patients with widespread malignancy but can also be the first indicator of an occult malignancy. ${ }^{2}$ Lung, renal, colon, and breast malignancies are known to be the principal primaries contributing to this condition, with $10.2 \%$ arising from breast primaries. ${ }^{3,4}$ Phyllodes tumours are fibroepithelial breast lesions that are predominantly benign but with possible malignant variants. Malignant variants have a reported incidence that ranges from $8 \%$ to $45 \%$ with a metastatic rate of $10 \% .^{10}$ Occurring in middle-aged women, they may be unifocal or multifocal.

Dissemination of malignant cells is postulated to occur via lymphatic spread. ${ }^{6}$ This was evident with the presence of cervical lymphadenopathy in our patient. The condition has a grave prognosis, with a mean life expectancy from the time of diagnosis of approximately 7 months ( \pm 7 months). ${ }^{3}$ The dominant hand is usually affected as it is perceived to receive a larger blood supply and more likely to sustain an injury compared to the non-dominant hand. ${ }^{5}$

It is theorized that trauma predisposes a patient to acrometastases, as trauma-induced tissue degradation permits tumour emboli to settle and proliferate within the bone. ${ }^{8}$ Alternatively, it is also thought that trauma results in a release of chemotactic factors that promote migration of tumour cells to bone. ${ }^{?}$

The middle finger is the most affected site followed by the thumb. Within the digit, these lesions are usually seen in the distal phalanx. ${ }^{6}$ Clinically, swelling, digital enlargement, mechanical dysfunction, pain, and occasionally erythema and ulceration are observed. Due to these features, clinicians may face a diagnostic conundrum as infections, inflammatory arthropathies, and metabolic derangements, such as gout, commonly mimic its appearance. This was apparent in our patient, as 2 separate physicians had misdiagnosed her with a fingertip infection before a correct diagnosis was made. The scenario of infection and acrometastasis occurring concurrently is theoretically possible; however, in our patient, the infection was excluded based on the radiographic and laboratory findings. Plain radiographs of the patient's left hand were suggestive of acrometastases as rabid obliteration (permeative lytic destruction) of bony architecture was visualized. This vastly differs from radiographic changes seen in osteomyelitis where periosteal elevation with a milder degree of osseous distortion is usually seen at this stage. Negative laboratory infective parameters further substantiated the patient's diagnosis.

\section{Conclusion}

Reports highlighting acrometastases masquerading as fingertip infections are scarce. Due to their rarity and manner of presentation, patients are commonly misdiagnosed. Poor prognosis and imminent death are associated with this condition, hence the need for a high index of suspicion to ensure prompt diagnosis and referral for palliative treatment.

\section{Conflict of interest}

The authors declare that there are no conflicts of interest.

\section{Funding}

This case report had no source of funding.

\section{Contribution of authors}

Collin Looi Seng Kim and Manohar Arumugam both diagnosed and managed the patient, and both contributed to the writing of the manuscript.

\section{How does this paper make a difference to general practice?}

- Increases awareness regarding acrometastases that may mimic fingertip infections

- Aids in identifying an occult malignancy, as acrometastases may be the first detectable sign of the primary pathology

- Highlights the need for prompt diagnosis and early referral for palliative care 


\section{References}

1. Kerin R. Metastatic tumors of the hand. J Bone Joint Surg Am 1983; 65: 1331-1335.

2. Abrahams TG. Occult malignancy presenting as metastatic disease to the hand and wrist. Skeletal Radiol 1995; 24: 135-137.

3. Afshar A, Farhadnia P, Khalkhali H. Metastases to the hand and wrist: an analysis of 221 cases. J Hand Surg Am 2014; 39: 923-932.

4. Stomeo D, Tulli A, Ziranu A, Perisano C, De Santis V, Maccauro G. Acrometastasis: a literature review. Eur Rev for Med and Pharmacol Sci 2015; 19: 2906-2915.
5. Healey JH, Turnbull AD, Miedema B, Lane JM. Acrometastases. A study of twenty-nine patients with osseous involvement of the hands and feet. J Bone Joint Surg Am 1986; 68: 743746.

6. Flynn CJ, Danjoux C, Wong J, Christakis M, Rubenstein J, Yee A, Yip D, Chow E. Two cases of acrometastasis to the hands and review of the literature. Curr Oncol 2008; 15: 51-58.

7. O’Sullivan ST, O'Donoghue JM, Hayes D, Shaughnessy M. Squamous cell carcinoma of the finger masquerading as an abscess. Scand J Plast Reconstr Surg Hand Surg 2000; 34:91-92.
8. Joll CA. Metastatic tumours of bone. Br J Surg 1923; 11: 38-72.

9. Tolo ET, Cooney WP, Wenger DE. Renal cell carcinoma with metastases to the triquetrum: case report. J Hand Surg Am 2002; 27: 876881.

10. Mangi AA, Smith BL, Gadd MA, Tanabe KK, Ott MJ, Souba WW. Surgical Management of phyllodes tumors. Arch Surg 1999; 134: 487492. 